ISSN: 1130-2887 - e-ISSN: 2340-4396

DOI: https://doi.org/10.14201/alh2019824362

\title{
EL IMPACTO DE LA INSTRUMENTALIZACIÓN DE ORGANIZACIONES LATINOAMERICANAS EN LA INTEGRACIÓN: TRES ILUSTRACIONES DESDE CHILE
}

The Impact of the Instrumentalization of Latin American Organizations on Integration: Three Illustrations from Chile

\author{
Sébastien DuBÉ \\ Universidad del Norte, Colombia \\ $\triangle$ sdube@uninorte.edu.co \\ Tatiana ZuluagA OROZCO \\ Consultora independiente, Chile \\ $\triangle$ zuluagaorozco@gmail.com
}

Fecha de recepción: 5 de enero de 2018

Fecha de aceptación y versión final: 22 de julio de 2019

RESUMEN: El artículo plantea que el uso político y estratégico de las organizaciones regionales por parte de los actores políticos, que son los gobiernos, las oposiciones y los grupos de la sociedad civil, contribuye a explicar las dificultades y la resiliencia de los procesos de integración latinoamericana. A través de la descripción de tres debates sociales y políticos que provocaron clivajes en Chile, propone mirar las lógicas políticas internas para entender la dinámica del regionalismo latinoamericano.

Palabras clave: organizaciones regionales; regionalismo latinoamericano; actores políticos; diplomacia de doble nivel; integración.

ABSTRACT: The article claims that the strategic and political use of regional organizations by political actors such as governments, opposition parties and social groups, contributes to the explanation of the weaknesses and the resilience of Latin American integration processes. Through the description of three political and social debates that provoked cleavages in Chilean society, it proposes to have a look at internal politics to understand the dynamics of Latin American regionalism.

Key words: regional organizations; Latin American regionalism; political actors; doubleedged diplomacy; integration. 
SÉBASTIEN DUBÉ Y TATIANA ZULUAGA OROZCO

\section{INTRODUCCIÓN ${ }^{1}$}

Desde el inicio del presente siglo, una amplia literatura sobre el regionalismo se desarrolla en torno al objetivo de explicar los fracasos, las dificultades o los estancamientos de los procesos de integración política y/o económica en América Latina. De manera general, los factores utilizados para explicar esos fenómenos son, primeramente, de carácter institucional o estructural. Entre otros, Dabène (2009) avanza que la debilidad de las instituciones nacionales es un factor que hace improbable que se vayan construyendo instituciones supranacionales fuertes en la región. Por otro lado, Malamud (2015) sostiene que el control que tiene el Poder Ejecutivo sobre las políticas exteriores y, por ende, sobre las decisiones relacionadas con el regionalismo explica la ausencia de organizaciones regionales fuertes con burocracias autónomas. Los trabajos que destacan las dificultades relacionadas con la multiplicación de organizaciones y de cumbres, el overlapping, y la fragmentación de la región también entran en los análisis de la arquitectura del regionalismo latinoamericano (Altmann Borbón 2011; Briceño Ruiz 2018; Mace et al. 2016; Nolte 2019; Weiffen 2017) y de sus particularidades institucionales.

Del lado de los argumentos estructurales, Guzman (1997), por ejemplo, retoma la lógica de la teoría de juegos para ilustrar cómo la estructura de las economías latinoamericanas dificulta la cooperación regional por la necesidad que tienen los Estados de atraer inversiones extranjeras para la explotación de sus recursos naturales. La doble lógica de cooperación y de competencia también está presente en las decisiones de participar en un alto número de organizaciones, según Shadlen (2008). Para ese autor, la lógica de competencia hace que los jefes de Estado perciban que la no participación en una organización puede representar un costo, dados los potenciales beneficios que los demás países podrían obtener de la cooperación. Según Cason (2011), el carácter periférico de la región en el sistema financiero internacional, acompañado de una falta de apoyo de las sociedades civiles, explica el estancamiento de las iniciativas de integración aun más exitosas como el Mercosur. Por otra parte, Legler (2011) señala que la región latinoamericana carece de un liderazgo fuerte favorable a la integración. Ese último fenómeno puede explicarse por el rechazo de otros países de ver a algún Estado acceder al «rango» de líder de la región (Wehner 2011), o simplemente por la falta de convicciones en el regionalismo latinoamericano.

1. La presente investigación fue iniciada mientras Sébastien Dubé era profesor en el Departamento de Historia de la Universidad de Santiago y fue terminada mientras se desempeña como profesor asistente en el Departamento de Ciencia Política y Relaciones Internacionales de la Universidad del Norte en Barranquilla, Colombia. Tatiana Zuluaga Orozco es consultora independiente en gestión de proyectos de cooperación internacional. Al inicio de la investigación era estudiante de la Maestría en Estudios Internacionales del IDEA (Santiago de Chile) y se desempeñaba como encargada de Relaciones Internacionales en el Instituto Nacional de la Juventud (INUJV) de Chile, cargo que ocupó de 2010 a 2017. Los autores agradecen los comentarios y las sugerencias de los dos evaluadores anónimos de América Latina Hoy, Revista de Ciencias Sociales, a la primera versión de este artículo. 
Irónicamente, la combinación de los factores mencionados conduce a una situación paradójica: la multiplicación de los tratados y organizaciones lleva a una «sobreoferta» de integración que resulta ineficiente por la dificultad de coordinar las acciones de numerosos organismos (Altmann Borbón 2011: 211). Esa sobreoferta también se ve ilustrada por una dinámica excesiva de «diplomacia de cumbres». Para demostrar ese punto, Portales (2016) ha contabilizado más de 300 cumbres internacionales involucrando a líderes latinoamericanos, organizadas entre los años 1992 y 2010, lo que significa estadísticamente una cumbre cada mes. Ahora, dichos encuentros suelen terminar con declaraciones muy optimistas en cuanto a sus supuestos resultados, en general desconectados de los alcances reales de las distintas iniciativas de integración en la región (Mace et al. 2016). La crisis actual del regionalismo latinoamericano parece haber reducido el ritmo de cumbres dada la paralización de organizaciones como la Comunidad de Estados Latinoamericanos y Caribeños (CELAC) y la Unión de Naciones Suramericanas (UNASUR), pero no ha significado la desaparición de ninguna de ellas hasta la fecha. Al contrario, la crisis de la UNASUR motivó a los mandatarios de Chile y de Colombia, Sebastián Piñera e Iván Duque, a proponer una nueva organización: el Foro para el Progreso y el Desarrollo de América Latina (PROSUR).

Paralelamente a los debates acerca de los factores que dificultan la integración latinoamericana, Dabène abre, a finales de la década pasada, una nueva línea de reflexión esta vez acerca de «la resiliencia de la agenda de la integración en la región a pesar de su inestabilidad y sus crisis» $(2009: 5)^{2}$. Como pistas de explicación, el autor sugiere considerar la importancia del aprendizaje histórico, los contextos políticos y económicos como catalizadores de olas de integración y la evolución de los objetivos de los Estados. Como complemento a dicha propuesta, Rivarola y Briceño (2013) plantean que la importancia de la autonomía regional, la búsqueda del desarrollo socioeconómico y la identidad supranacional latinoamericana son tres factores que explican la vigencia de la agenda de integración en la región, prácticamente sin interrupción desde las independencias de inicios del siglo XIX (Rivarola Puntigliano y Briceño Ruiz 2013). De alguna manera, las respuestas ofrecidas para esa nueva agenda de investigación de la resiliencia de los procesos de integración en América Latina mantienen las lógicas institucionalistas y estructuralistas mencionadas anteriormente.

El presente artículo aborda la problemática de las dificultades y de la resiliencia de los procesos de integración en América Latina desde una mirada enfocada en los actores políticos. Como complemento a los estudios enfocados en las organizaciones regionales y en las relaciones interestatales entre los países de la región, el artículo plantea que las dinámicas políticas internas desincentivan el abandono de las organizaciones regionales, pero sin incentivar la profundización de los procesos de integración. Precisamente, el planteamiento principal es que la estructura del regionalismo latinoamericano facilita

2. Todas las citas provenientes de textos originales escritos en otro idioma que el español son traducciones libres de los autores del presente artículo. 
la posibilidad, por parte de los distintos actores políticos internos en competencia, de utilizar las organizaciones regionales en función de sus intereses particulares y cortoplacistas. Por actores políticos, se consideran tres categorías: (1) el gobierno de turno y los partidos que lo apoyan en la totalidad de su programa o en áreas específicas; (2) los partidos, líderes o movimientos que forman la oposición al gobierno, y (3) los distintos grupos que pertenecen a la sociedad civil.

De esa manera, el análisis propuesto no se orienta directamente hacia el funcionamiento de las organizaciones regionales como tal, sino hacia la dinámica política interna de un país, la cual involucra a una o a varias organizaciones. Así, se puede proponer una lectura de la relación entre los Estados y las organizaciones regionales que hace eco a la necesidad de superar la separación entre la política interna y la política exterior, como lo sugiere Hill (2003). Además, la perspectiva adoptada aplica explícitamente la lógica clásica según la cual la diplomacia es un juego de «doble nivel» (Evans et al. 1993; Moravcsik 1993a) y una dinámica que involucra varios niveles y actores (Aguirre Azocar y Erlandsen 2018; Cooper et al. 2013). Lo anterior lleva a plantear que los cálculos estratégicos de los actores políticos en la política interna afectan la política exterior hacia los procesos de integración regional y, al revés, las organizaciones regionales impactan en la política interna al ofrecerles recursos a unos u otros actores para el avance de sus agendas particulares. Finalmente, el argumento central es que los actores políticos no solo apoyan la integración para el alcance de «intereses estatales», como el crecimiento económico y el desarrollo, sino que en algunas circunstancias instrumentalizan los recursos que proveen los procesos de integración y los organismos regionales para su propio beneficio político. Antes de entrar más en detalle con respecto a las bases conceptuales y teóricas que enmarcan el argumento y el análisis, es necesario aclarar las definiciones utilizadas para referirse a integración, regionalismo y organizaciones regionales.

Dado que el enfoque principal del artículo corresponde a las relaciones triangulares que existen entre los actores políticos internos que utilizan las organizaciones regionales en el contexto latinoamericano, una definición amplia de la integración regional como la que emplea Dabène se revela útil. Según este autor, la integración es un proceso de «incremento de las interacciones entre unidades políticas provistas por actores que comparten ideas en común, determinan objetivos y definen métodos de lograrlos, contribuyendo así a la construcción de una región» (2009: 10-11). También, Dabène advierte que esa definición se caracteriza por «tratar de cooperación internacional y de toma de decisión colectiva, lo que hace legítimo el ejercicio de tratar de identificar a los actores involucrados e indagar sus motivaciones, los métodos que ocupan y los objetivos que buscan» (2009: 7-8). Vistos así, los procesos de integración política y/o económica y la creación de organizaciones regionales forman parte de la dinámica definida como el proceso de regionalismo. Como lo sugiere Tussie, la noción de regionalismo combina elementos culturales e históricos, canales de difusión de políticas, coordinación de estrategias y de objetivos entre gobiernos (Tussie 2009: 169-170). Por «organizaciones regionales» se entiende los acuerdos, las normas y los tratados de los cuales varios o la totalidad de los Estados de la región forman parte. Más concretamente, dado que los temas analizados conciernen a las disputas internas en Chile con respecto a derechos humanos y sociales, las principales organizaciones regionales a las cuales el artículo se refiere son las que conforman el Sistema Interamericano de Derechos Humanos. 


\section{PRECISIONES TEÓRICAS Y CONCEPTUALES}

\section{II.1. Intergubernamentalismo revisado, actores y desconfianza}

Con respecto al enfoque en los actores, el artículo retoma, pero adapta, la lógica del intergubernamentalismo liberal de Moravcsik (1993b) para postular e ilustrar que las políticas exteriores son el resultado de procesos políticos internos que involucran a una variedad de actores defendiendo sus intereses particulares. Sin embargo, dos matices son avanzados. En primer lugar, el artículo trata los «actores» como unidades formadas por individuos. Es decir, retoma las ideas de Meyer (2010), que enfatiza que las «organizaciones y los Estados son concebidos como actores derivados de sus miembros como individuos» (Meyer 2010: 2), lo cual lleva a considerar a las personas -y no solo las funciones o los cargos-. En ese sentido, el enfoque se acerca a las perspectivas analíticas propias de la toma de decisión en materia de política exterior que consideran la importancia de los cálculos estratégicos, las ideologías, los objetivos personales y las personalidades de los líderes (Hermann 2001; Hudson 2007). En segundo lugar, a diferencia de la propuesta de Moravcsik, el análisis no se limita a la jefatura del Estado, sino que se aplica también a los grupos que conforman la oposición y los movimientos sociales.

Dos razones explican la postura teórica de considerar los tres tipos de actores y no solamente a los jefes de Estado. Primero: a partir del planteamiento según el cual la política exterior liderada por el o la mandatario/a resulta de cálculos y de conflictos internos, se postula que los actores contrarios al gobierno mantienen su postura y sus propios objetivos en materia de política interna y exterior. Es decir, si bien es cierto que las políticas exteriores pueden ser vistas como menos sujetas a disputas partidistas por ser «políticas de Estado» o porque suscitan menos interés por parte de políticos enfocados hacia lo nacional y lo local, siguen siendo políticas públicas. Es más, las políticas exteriores latinoamericanas dependen de consideraciones tanto pragmáticas como ideológicas (Gardini y Lambert 2011), y sus «macro orientaciones», como la decisión de perseguir un mayor acercamiento hacia los países del norte o de buscar una mayor autonomía regional, también dependen de la ideología del gobierno de turno (Merke y Reynoso 2016). En ese contexto, la oposición de grupos de la sociedad civil a las orientaciones de las políticas sociales, por ejemplo, puede trasladarse naturalmente a la esfera de la política exterior.

Segundo: una particularidad contextual latinoamericana -pero no necesariamente exclusiva a la región-invita a considerar a la oposición y a los movimientos sociales como actores que pueden utilizar el escenario regional para sus objetivos particulares cuando estos van en contra de los objetivos del gobierno. La insatisfacción elevada con el funcionamiento de la democracia en América Latina y los bajos niveles de apoyo y de confianza en los gobiernos y en las instituciones pueden incentivar las estrategias de la oposición y de la sociedad civil a la instrumentalización de las organizaciones regionales en favor de sus intereses y para debilitar a los gobiernos. Según el informe de Latinobarómetro para el año 2018, apenas un 24\% de latinoamericanos afirma estar satisfecho con la democracia, el nivel más bajo desde las primeras mediciones de la corporación en 1995 (Latinobarómetro 2018: 35). Aún más baja (17\%) es la proporción de los individuos que están de acuerdo con la afirmación de que «Se gobierna para el bien de todo el pueblo» 
(2018: 40). En cuanto a la confianza en las instituciones, los partidos políticos obtienen un $13 \%$ de respaldo, el Congreso un $21 \%$ y el gobierno solo un $22 \%$.

La desconfianza no tiene solo como impacto el crear un distanciamiento entre la sociedad civil y las autoridades, sino que, en el contexto latinoamericano, el mismo Estado puede ser visto como un actor que viola los derechos de sus propios ciudadanos. Por ejemplo, los datos de la Comisión Interamericana de Derechos Humanos (CIDH), principal organismo regional que ofrece una posibilidad a los latinoamericanos de demandar a su Estado, señalan que la organización recibió 2.494 peticiones durante el año 2017 y que tiene más de 4.000 peticiones vigentes, pendientes de su estudio inicial (CIDH 2019). En ese contexto regional, la lógica de intergubernamentalismo liberal mencionada anteriormente debe ser contextualizada. En América Latina, una sociedad civil contraria al gobierno puede, estratégicamente, apoyar una política exterior a favor de la integración regional si percibe que le puede dar recursos para presionar al gobierno o defenderse del mismo Estado.

\section{II.2. Factores individuales y sistémicos}

Dado que el análisis se enfoca en el comportamiento de actores que buscan recursos a nivel regional para obtener ganancias en un escenario político nacional, el artículo combina elementos de relaciones internacionales y de política interna. Por ganancias, se considera cualquier elemento material o no material que permita a un actor defender y promocionar sus intereses y su agenda. En el caso de los individuos que forman el gobierno, se considera la capacidad de mantenerse en el poder, ganar una futura elección y/o mantener su tasa de aprobación lo más elevada posible. En el caso de la oposición, el principal interés consiste en acercarse al poder promoviendo su propia agenda y buscando la debilidad del gobierno. En el caso de los grupos de la sociedad civil, se considera el avance de la agenda o la misión particular que tienen y motiva su existencia.

Otras dos perspectivas teóricas claves sirven de base para explicar el argumento presentado y justificar su pertinencia. La primera es la lógica de la teoría de roles, la cual busca conectar el análisis de las políticas exteriores con las relaciones internacionales. Aplicado en el ámbito de las relaciones internacionales, ese modelo tiene la ventaja de poder combinar elementos sistémicos propios de la dinámica internacional con elementos individuales relacionados con los determinantes de la toma de decisión.

Para Walker, la conexión entre los procesos de toma de decisión y el sistema internacional se hace, conceptual y teóricamente, vía la línea investigativa del análisis de los comportamientos de relaciones internacionales («behavior international relations») (Walker 2011: 4). El interés de ese modelo es que, a diferencia de las perspectivas realistas o constructivistas, este introduce una serie de factores de carácter sociológico y psicológico que deben ser considerados al momento de analizar las decisiones que toman los actores en materia de política exterior.

Dicho modelo plantea que, gracias a la teoría de roles, se pueden analizar las dinámicas de conflictos y de cooperación a través de la conexión de las «microdecisiones de los actores en materia de política exterior», correspondientes a su percepción del mundo, 
con las realidades macro del «mundo de los eventos» (Walker 2011: 6). Esta combinación permite, tanto a nivel regional como a nivel internacional, observar las «creencias generadas por los procesos cognitivos, emocionales y motivacionales de los líderes [...] y los eventos reales generados por la presencia, el poder y el comportamiento de otros actores» (ibid. $)^{3}$. En el análisis de las políticas exteriores latinoamericanas y de su impacto en los procesos de integración en la región, Wehner (2015) y Wehner y Thies (2014) han demostrado la validez de este modelo para analizar las relaciones entre los Estados. Para la presente investigación se busca aplicar la misma lógica, pero entre los actores internos, considerando así los objetivos de cada uno, sus decisiones, sus declaraciones, sus estrategias y cómo estas se ven afectadas por el sistema en el cual se sitúan. En este caso, por «sistema» se entiende el sistema político interno, pero también las organizaciones regionales involucradas en las temáticas que generan conflictos políticos internos.

La última referencia teórica de interés para este artículo combina elementos de identidad con la transformación del sistema internacional. En un contexto en el cual la satisfacción y la confianza en las instituciones políticas se encuentran bajas, el sistema internacional globalizado puede cumplir un papel de fuente diversa de apoyo para los actores descontentos con el actuar de los gobiernos nacionales. Aquí, un aporte conceptual propuesto por Scholte (2016) que facilita la comprensión del actuar de los grupos de la sociedad civil en ese entramado político nacional e internacional es el de la «gobernanza policéntrica». El punto de partida del concepto de Scholte es la observación de un proceso de alteración de las geografías y de las identidades políticas (2016: 739). Dicho proceso lleva a la instauración de un nuevo modo de gobernanza, que el autor define como «policéntrico» y que tiene siete características fundamentales. De ellas, las significativas para el presente análisis son que la nueva gobernanza implica: (1) políticas e instituciones de distintos niveles, y no únicamente el nivel estatal; (2) una regulación difusa y no centralizada que involucra varias entidades; (3) la superposición de varias jurisdicciones y (4) la ausencia de jerarquías claras (Scholte 2016: 739).

En términos concretos, el modelo de Scholte permite explicar la multiplicación de opciones y de estrategias que quedan a la disponibilidad de los grupos sociales para alcanzar sus objetivos. El proceso de erosión de la lealtad o de la identidad hacia el Estado nacional, acompañado de la multiplicación de los niveles de toma de decisión o de las jurisdicciones, abre oportunidades de acción para los grupos sociales. Para promover sus intereses y agendas no solo tienen la opción de presionar al gobierno de turno: pueden aliarse a organizaciones internacionales y/o a la oposición, involucrando jurisdicciones o normas que no existían antes. Es más, cuando las reivindicaciones de los grupos sociales tocan elementos de carácter identitario -como la pertenencia a una comunidad étnica, lingǘstica o de género minoritaria- puede ocurrir el fenómeno descrito por Adler: «La gente empieza a imaginar que comparte su destino con personas de otros países que comparten sus valores y sus expectativas de comportamiento en los asuntos políticos nacionales e internacionales» (1997: 380).

3. Las cursivas son del texto original. 
La combinación de las perspectivas teóricas mencionadas acerca de los comportamientos de relaciones internacionales con la idea de «gobernanza policéntrica» tiene por lo menos cuatro consecuencias destacables para la conducción del presente análisis. Primero: se sostiene que los principales actores políticos dentro de un Estado no tienen una postura consensuada en relación con la integración y que la política exterior puede ser el objeto de mayores discusiones y dinámicas de politización de lo que la literatura plantea. Segundo: la integración puede ser un medio para alcanzar metas políticas más que un fin en sí. Tercero: dichas metas pueden ser particulares y no nacionales. Cuarto: las motivaciones originales de la inserción internacional de los Estados importan para entender las decisiones de los gobiernos, pero pueden llevar a efectos no deseados por estos últimos. Esas cuatro consecuencias serán demostradas en el análisis de tres temáticas que han marcado la agenda política chilena principalmente desde el inicio de la década de los 2000.

\section{METOdOLOGÍA Y ANÁLISIS: LA ELECCIÓN DE CHILE Y DE TRES TEMAS OBSERVADOS}

\section{III.1. El caso de Chile}

$\mathrm{El}$ análisis de la siguiente sección se divide en tres ejemplos que sirven para ilustrar cómo la integración regional en América Latina otorga recursos para que distintos actores políticos puedan promover sus propios intereses en el escenario político nacional. Los tres ejemplos se relacionan con debates sociopolíticos que han marcado la vida política chilena durante las últimas dos décadas. El interés de elegir a Chile es doble. Por un lado, tal como será explicado a continuación, los gobiernos chilenos, principalmente desde 1990, han promovido la participación activa de Chile en un gran número de tratados y organizaciones regionales e internacionales. Además, la cuestión de la internacionalización y del respeto a los tratados y compromisos internacionales ha tomado un lugar central en la política exterior del país (MINREL 2017). Por otro lado, la estrategia de inserción de Chile ha seguido una lógica «pragmática» más que «ideológica», abriendo así la puerta a dinámicas políticas internas conflictivas y multiplicando los recursos para los distintos actores que compiten en la escena política nacional. En principio, se puede recordar, como lo sostiene Fuentes (2011), que las élites políticas chilenas del periodo postdictadura iniciaron una estrategia de inserción global, firmando varios tratados y convenciones para legitimar el Estado de Chile en el escenario internacional después de los años de aislamiento diplomático durante el régimen de Pinochet (Fuentes 2011: 20). Por esos motivos, el Estado de Chile integró una serie de organismos internacionales y adoptó varias normativas relacionadas con temáticas políticas y sociales a inicios de los años 1990. Por ejemplo, durante el gobierno de Patricio Aylwin (1990-1994), Chile simbolizó su «retorno a la comunidad internacional» adoptando la Convención de las Naciones Unidas sobre los Derechos del Niño (1990), la Convención Americana sobre Derechos Humanos (1991) y la Convención 144 de la Organización Internacional 
del Trabajo (1992) ${ }^{4}$. A esos tratados enfocados en temáticas sociales y políticas muy a menudo relacionadas con los derechos humanos, siguió el ingreso en un alto número de tratados comerciales y económicos, particularmente durante el gobierno de Eduardo Frei (1994-2000).

En el contexto del presente artículo, el origen y los motivos de la estrategia de inserción internacional de Chile que llevó a adherir a varias organizaciones y tratados son elementos clave. Aunque no es necesariamente único, el caso de Chile es interesante dado que esa inserción internacional se hizo a pesar del conservadurismo de la sociedad chilena (Garretón y Garretón 2010: 131-132) y del carácter insular de la misma (Fuentes 2011: 20). Por lo mismo, Fuentes caracteriza dicha estrategia como «aspiracional», en la medida que se buscaba «integrarse a un mundo distinto de la realidad del país». Que se haya buscado la integración a la región y al mundo por motivos comerciales, pero también ideológicos y simbólicos, demuestra que la política exterior chilena ha sido instrumentalista en lo social y en lo económico. El análisis de tres debates nacionales relacionados con temáticas sociales y morales permitirá ilustrarlo, con sus consecuencias, con más precisión.

\section{III.2. Tres temas y dinámicas de instrumentalización de organizaciones regionales}

Para demostrar la validez del argumento planteado, la siguiente parte describe tres debates políticos y sociales que marcaron la agenda política nacional de los gobiernos de Michelle Bachelet (2006-2010), Sebastián Piñera (2010-2014) y/o Michelle Bachelet (2014-2018). Son tres temáticas que involucran políticas públicas nacionales que causan clivajes sociales y que se relacionan con tratados, normas o convenciones internacionales de los cuales el Estado de Chile es firmante. Más que entrar en el detalle de cada debate, lo que se pretende hacer es destacar las estrategias reales o discursivas de los actores que utilizan recursos provistos por el regionalismo latinoamericano en sus conflictos políticos en el escenario nacional chileno.

La primera temática es la política de prevención del embarazo adolescente que Michelle Bachelet impulsa en su primer gobierno (2016-2010) al recurrir a programas de la Comunidad Andina de Naciones (CAN), organización a la cual Chile se integró como miembro asociado en 2006. Este ejemplo ilustra la oportunidad que representa una organización internacional para que un gobierno pueda implementar una política pública que provoca resistencias internas. La segunda temática corresponde a la instrumentalización de la Comisión Interamericana de Derechos Humanos (CIDH) por parte de la oposición chilena con respecto a la problemática de las condiciones en las cuales viven los menores bajo la supervisión del Servicio Nacional de Menores (SENAME), esta vez durante la segunda administración Bachelet (2014-2018). Finalmente, el tercer caso analizado es el actuar del Movimiento de Integración y Liberación Homosexual (MOVILH)

4. El listado completo de los tratados internacionales firmados por el Estado chileno se encuentra aquí: www.bnc.cl. 
en su campaña para que las autoridades chilenas modifiquen la ley que regula el matrimonio, a partir de un fallo de la Corte Interamericana de Derechos Humanos. Como se podrá observar, el MOVILH recurrió frecuentemente a las instancias interamericanas para presionar al gobierno desde la segunda mitad de la década pasada.

\section{III.3. El uso del regionalismo por el gobierno para imponer una política polémica}

La salud sexual adolescente toma su lugar como temática relevante en las agendas nacionales latinoamericanas en general, y chilena en particular, en la década de los 2000 a partir de diagnósticos que evidenciaban que América Latina era la única gran región del mundo en la cual la tasa de fecundidad adolescente había aumentado entre 1980 y 2000 (Rodríguez Vignoli 2008: 16). En los mismos años, la Comisión Económica para América Latina y el Caribe (CEPAL) relaciona el embarazo adolescente con problemáticas de embarazo no deseado, riesgos de salud para las madres, iniciación sexual temprana o falta de educación sexual. Además, se plantea la necesidad de adoptar políticas públicas de salud sexual y de acceso a derechos sexuales y reproductivos para una mayor igualdad de oportunidades (Hopenhayn 2008). La formulación de dichas recomendaciones sigue los lineamientos en materia de salud sexual y reproductiva y de derecho a educación sexual para los adolescentes, planteados en la Conferencia Internacional sobre Población y Desarrollo de El Cairo de 1994 y en la Cuarta Conferencia Mundial de la Mujer en 1995 en Beijín, ambas adheridas por Chile (Dides et al. 2011; MINSAL 2001).

En el Chile postdictatorial, la transición hacia un nuevo marco paradigmático en el cual se relaciona sexualidad-reproducción con derechos sexuales y reproductivos es un proceso arduo, fuertemente opuesto por amplios sectores ubicados a la derecha del espectro político y cercanos a la Iglesia católica (Araujo 2009: 17-18). Para contextualizar la influencia de dichos sectores en la sociedad y en las políticas públicas, se puede mencionar que el Estado de Chile otorgó la igualdad de derechos entre los hijos nacidos de padres casados y no casados apenas en 1995, y que fue solo en el año 2004, después de 10 años de debates legislativos, que se legalizó el divorcio (Quilodran 2004). La importancia de la oposición de ideología conservadora, tanto en la política como en la sociedad civil, también explica que, hasta el año 2017, Chile fue uno de los pocos países democráticos en prohibir el aborto en caso de feto inviable, de riesgo vital para la mujer embarazada o de embarazo producto de una violación.

Es en ese contexto que, en 2005, el presidente socialista Ricardo Lagos (2000-2006) promueve la iniciativa gubernamental para el acceso a anticoncepción de emergencia (o píldora del día después) a toda mujer mayor de 14 años, a través de su establecimiento en las Normas Nacionales de Regulación de Fertilidad del Ministerio de Salud (MINSAL). La primera aprobación de un fármaco similar por parte del Instituto de Salud Pública en 2000 está, en ese momento, bloqueada, y lo estará hasta 2009 (Molina 2013: 79). La política del gobierno Lagos suscita las críticas de sectores de la oposición parlamentaria (Alianza por Chile) y del ala más conservadora de la Concertación, quienes consideran el acceso al fármaco como una medida abortiva. Es así que, en el año 2006, parlamentarios opositores al gobierno de Michelle Bachelet (2006-2010), el cual busca seguir 
en la línea de la administración anterior, presentan una acción judicial ante el Tribunal Constitucional solicitando declarar inconstitucionales las normas que contemplaban la distribución de la anticoncepción de emergencia. El clivaje político y social alrededor del acceso a píldoras anticonceptivas de emergencia es tal que, a lo largo de la década, «el tema estuvo en forma permanente en todos los medios de comunicación, tanto nacional como internacional» (Molina 2013: 79).

El uso de organizaciones regionales para lograr objetivos políticos particulares se ilustra con la adhesión de Chile como miembro asociado de la CAN (ex Pacto Andino, del cual Chile fue miembro desde su fundación en 1969 hasta su salida en 1976) en 2006. Aquí vale la pena recordar que la decisión de las autoridades chilenas de reingresar a dicha organización se produce mientras la misma atraviesa una crisis profunda. Irónicamente, la firma de los Tratados de Libre Comercio entre Perú y Estados Unidos en 2005 y entre Colombia y Estados Unidos en 2006 provocó la salida de Venezuela de la CAN el mismo año que Chile oficializa su regreso. Es más, la crisis de la CAN llevó al entonces presidente peruano Alan García a proponer una nueva alternativa de integración en 2007, el Arco del Pacífico -que se transformará posteriormente en la actual Alianza del Pacífico-y de la cual el gobierno chileno fue un miembro activo desde el inicio.

La agenda y el marco del ingreso de Chile a la CAN se definieron en la Decisión 666 de la Comisión Mixta Chile-CAN encargada de definir las esferas de cooperación entre las partes. Si bien dicho documento evoca una agenda de trabajo amplia que también incluye temas como la energía y el comercio, es llamativo que el listado de las «materias de interés recíproco» mencionen el desarrollo humano y social incluyente y sobre todo «las prioridades establecidas en el Organismo Andino de Salud-Convenio Hipólito Unanue» (CAN 2007: art. 2.d.).

La nueva participación del Estado chileno en las instancias de salud de la CAN mencionadas le permitió al gobierno avanzar en su agenda en materia de política de educación sexual y reproductiva hacia los adolescentes, un objetivo contenido en el programa de gobierno de Michelle Bachelet para la elección presidencial de 2005 (Bachelet 2005: 89). En 2007, los ministros de Salud de la CAN, agrupados en el Organismo Andino de Salud,

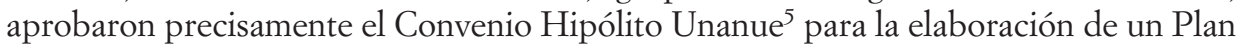
Andino de Prevención del Embarazo en Adolescentes (PLANEA) bajo la conducción del Comité Subregional Andino y con el respaldo de distintas organizaciones internacionales (PLANEA 2007). El mandato que la CAN otorga al PLANEA consiste en «disminuir las brechas que dificultan el acceso a los servicios de salud por parte de la población adolescente, promoviendo el ejercicio de los derechos humanos, incluidos los sexuales y reproductivos» (ibid.). El objetivo se alinea con la agenda del gobierno Bachelet en materia de salud sexual, la cual está fuertemente resistida por la oposición y la sociedad civil más conservadora del país.

5. El Organismo Andino de Salud se define como un organismo de integración subregional, perteneciente al Sistema Andino de Integración, cuyo objetivo es coordinar y apoyar los esfuerzos que realizan los países miembros, individual o colectivamente, para el mejoramiento de la salud de sus pueblos: http://www.orasconhu.org/quienes-somos/presentaci\%C3\%B3n. 
Meses después de la adopción del Convenio Hipólito Unanue, el gobierno Bachelet promulgó la «Política Nacional de Salud de Adolescentes y Jóvenes 2008-2015», cuya definición estaba en directa relación con los acuerdos adquiridos en el marco del PLANEA. La implementación de distintas medidas en ese marco también llevó a la adopción en 2010 de la Ley 20.418, después de más de tres años de trámite legislativo, sobre la prevención del embarazo adolescente, la educación sexual y la anticoncepción de emergencia.

El ejemplo anterior recuerda un planteamiento de Moravcsik, según el cual los «gobiernos nacionales tienen un interés en cooperar cuando la coordinación de políticas aumenta su control sobre los resultados de las políticas públicas nacionales, permitiendo el alcance de objetivos imposibles por otras vías» (Moravcsik 1993b: 485). Claramente, es difícil comprobar que, sin el ingreso a la CAN y a sus instancias en materia de salud, hubiese sido imposible que el gobierno Bachelet implementara sus políticas en materia de reducción del embarazo adolescente, acceso a fármacos anticonceptivos y educación sexual. También sería excesivo avanzar que el ingreso de Chile a la CAN fue principalmente motivado por la agenda del gobierno Bachelet en temas sociales. Sin embargo, existe evidencia de que la participación de Chile en la organización fue clave para el establecimiento de marcos legales progresistas, por parte del gobierno, acerca de temáticas fuertemente resistidas por otros actores políticos de ideología más conservadora.

\section{III.4. El uso de las organizaciones internacionales por la oposición para presionar al gobierno}

La instrumentalización de organizaciones internacionales por partidos de oposición para enfrentar a un gobierno es, en sí misma, más sencilla de observar. Considerando las limitaciones institucionales que pueden tener las oposiciones para obstaculizar a los gobiernos en la implementación de sus agendas, el regionalismo puede ofrecer recursos reales o discursivos para las discusiones políticas internas. Si las organizaciones y tratados que conforman el regionalismo tienen algún grado de poder vinculante en un área específica, la oposición puede recurrir a ellos para avanzar en su agenda y/u obstruir la ejecución de la del gobierno.

Aquí, el ejemplo concreto para ilustrar esta dinámica se refiere a una estrategia político-comunicacional de la oposición de derecha al segundo gobierno de Bachelet en el contexto de una crisis en el Servicio Nacional de Menores (SENAME). En Chile, el SENAME es un organismo público creado en 1979 que depende del Ministerio de Justicia y Derechos Humanos. Su misión incluye la protección de los derechos de los niños y adolescentes vulnerados y la reinserción social de menores infractores de la ley ${ }^{6}$. Gestiona distintos programas e instalaciones, incluyendo una unidad de adopción y hogares para cuidar y proteger a los menores en situación vulnerable.

Durante el año 2016, la muerte de una menor de 11 años en un centro del SENAME de Santiago desató una crisis política al interior del oficialismo no solo por la gravedad de los acontecimientos, sino por la falta de claridad en las explicaciones y respuestas de las

6. Véase : www.sename.cl. 
autoridades. Distintas investigaciones periodísticas revelaron la existencia de una crisis de proporciones mayores, cuestionando la estructura misma del sistema. En ese contexto, se sumaron las denuncias de funcionarios del SENAME con respecto a las condiciones de hacinamiento, el mal estado de los centros, la falta de personal y la ausencia de procedimientos coordinados de atención a menores en el sistema de salud. También se denunció el uso y el abuso de psicofármacos en los hogares del SENAME, los cuales se administran a los menores para evitar comportamientos disruptivos que puedan poner en riesgo su integridad. Finalmente, se destacó que el Estado de Chile no cumplía de pleno con las normas internacionales al proporcionar un funcionario por cada treinta niños en sus centros, lejos de la norma internacional de un educador por cada siete menores (Albert y Sepúlveda 2016).

A raíz de lo acontecido y de las declaraciones contradictorias de autoridades del Ministerio de Justicia, la Cámara de Diputados solicitó una comisión especial de investigación. Paralelamente al trabajo de la comisión, las autoridades del SENAME informaron que, entre los años 2005 y 2016, un total de 865 menores bajo la responsabilidad del organismo habían fallecido, cifra que subió a 1.313 en un nuevo informe difundido en julio de 2019 (El Mercurio 2016; Sepúlveda y Guzmán 2019). En cuanto al caso que desató la crisis, las investigaciones revelaron que el fallecimiento de la menor había sido causado por ahogamiento a manos de dos cuidadoras que intentaban «tranquilizarla», y no al uso de medicamentos tal como las autoridades lo habían declarado previamente.

La magnitud de la crisis y las conductas de las autoridades gubernamentales permitieron a la oposición utilizar el caso para atacar al gobierno por su gestión del SENAME y también instrumentalizar a una organización regional, la Comisión Interamericana de Derechos Humanos (CIDH), en su acusación de incumplimiento de los compromisos internacionales del Estado chileno. A finales del año 2016, diputados de la oposición ingresaron un requerimiento a la CIDH, la cual cuestionó en su sesión del 24 de mayo 2017 el hecho de que Chile es uno de los pocos países de América Latina que no cuenta con legislación apropiada para la protección integral de la infancia.

Si bien se podría argumentar que los diputados de la oposición actuaron por motivos distintos a consideraciones estrictamente políticas en este caso, es necesario destacar que una investigación realizada durante la presidencia del líder de la coalición de derecha, Sebastián Piñera (2010-2014), ya había denunciado las condiciones de vida de los menores en los centros del SENAME. En 2013, la Comisión Jeldres, realizada en conjunto por el Poder Judicial y la UNICEF, había revelado la existencia de problemas tales como vulneración de derechos, prostitución, violencia física y verbal, medicación y abuso sexual en los hogares del SENAME. Frente a una situación cuyas características incluían, pero eran anteriores al segundo gobierno de Bachelet (2014-2018), los mismos diputados que pidieron la intervención de la CIDH buscaron que se limitase la investigación al SENAME al periodo 2014-2017, para evitar considerar su gestión del organismo durante el periodo 2010-2014.

Lo interesante del presente caso es que, dado que la situación de negligencia del Estado es de larga data, los opositores de derecha critican a los gobiernos de izquierda, y los opositores de izquierda critican a los gobiernos de derecha con la misma retórica 
por la gestión de los centros del SENAME. Obviamente, eso no es de sorprender en la medida que es de la naturaleza misma de la disputa política. Sin embargo, lo irónico es la tendencia que tienen las oposiciones a querer involucrar a las organizaciones internacionales para investigar y difundir informaciones para luego querer limitar el quehacer de las mismas una vez que estén en el poder. En 2016, dos diputados de la oposición (de derecha) solicitaron el involucramiento de la CIDH para investigar la situación en los centros del SENAME (Carreño 2016). En 2018, fue el turno de congresistas de oposición de izquierda de solicitar lo mismo, esta vez en un centro de la región de Valparaíso (ADN 2018). En cada situación, el gobierno de turno, tampoco es de sorprender, busca relativizar las críticas de las organizaciones internacionales (Cerna 2018; Guzmán 2013; Roa 2018; Sepúlveda y Guzmán 2019). Es más, es llamativo que aquellas fuerzas políticas que pedían la intervención de la CIDH en Chile en 2016 son de la misma coalición que, ahora en el gobierno, están promocionando una reforma del Sistema Interamericano de Derechos Humanos con la finalidad de limitar sus poderes y el alcance de sus fallos (García 2019).

\section{III.5. El uso de las organizaciones internacionales por grupos de la sociedad civil para presionar al gobierno o a la oposición}

El Movimimiento de Integración y Liberación Homosexual (MOVILH) es una organización creada en 1991 para defender los derechos de las minorías sexuales en Chile. Por larga data, la adopción de una ley permitiendo el matrimonio entre dos personas de mismo sexo ha estado en el centro de sus reivindicaciones. En el sistema legislativo, la primera iniciativa fue presentada en 2008 por diputados oficialistas durante el primer mandato de la presidenta Bachelet. Después de años de estancamiento, un nuevo proyecto de ley fue presentado ante el Congreso en 2017, hacia finales del segundo gobierno Bachelet. Al momento de redactar este artículo, un año y medio después, dicho proyecto sigue en discusión en el Congreso (Iguales 2019).

La reivindicación del MOVILH tomó una dimensión internacional concreta en el año 2012 cuando la Corte Interamericana de Derechos Humanos falló a favor de la ciudadana chilena y jueza Karen Atala Riffo. La Sra. Atala había perdido la custodia de sus hijas a raíz de un juicio de la Corte Suprema chilena en 2004, decisión justificada por la orientación sexual de la denunciante. En su fallo, la Corte Interamericana declaró al Estado chileno responsable de discriminación en contra de la mujer -lesbiana y viviendo con su pareja- y de interferir de manera arbitraria en la vida privada y familiar de la mujer.

El fallo abrió la puerta a una potencial revisión de las legislaciones chilenas. Durante la campaña electoral de 2013, para un segundo mandato, la coalición liderada por Bachelet se comprometió a revisar la ley existente y, a partir de un debate público, a enviar un proyecto de ley de matrimonio igualitario (Nueva Mayoría 2013: 109). Sin embargo, la falta de avances en la materia y las divisiones dentro de la coalición gobernante sobre el tema motivaron el reclamo del MOVILH ante la CIDH por la falta de cumplimiento de las obligaciones internacionales del Estado chileno frente a las minorías sexuales. La Comisión acogió la solicitud del MOVILH y decretó la obligación del Estado chileno de iniciar nuevas discusiones con el MOVILH. La resolución de la CIDH, además, indicaba 
que un nuevo incumplimiento del Estado obligaría a la misma Comisión a definir un plazo para forzar a las autoridades chilenas a modificar las leyes vigentes.

Si bien el calendario electoral hizo que el gobierno dilatara el debate acerca del matrimonio igualitario para después de las elecciones municipales del año 2016, las acciones del MOVILH con la CIDH obligaron al gobierno a reponer el tema de la revisión de la ley en la agenda. Es en esas condiciones que se presentó el nuevo proyecto de ley en 2017, habiendo dado las presiones de la $\mathrm{CIDH}$ a Bachelet un espacio de poder frente a la oposición interna y externa al matrimonio igualitario. Es más, la retórica del gobierno para tratar de imponer una nueva ley explícitamente destaca que dicha legislación correspondía a «una obligación internacional» para las autoridades estatales (Gobierno de Chile 2017).

\section{CONCLUSIONES}

La literatura «clásica» sobre el regionalismo latinoamericano suele enfocarse en las decisiones de los jefes de Estado, las cuales son limitadas o influidas principalmente por factores institucionales o estructurales. Dicha perspectiva permite entender de manera muy general los problemas constantes de los procesos de integración latinoamericana en cuanto a sus resultados limitados o a la debilidad de su institucionalidad. De manera complementaria a esta amplia literatura, este artículo plantea que la debilidad de la integración latinoamericana puede explicarse por la instrumentalización, es decir, el uso estratégico que los actores políticos hacen de las organizaciones regionales para la promoción de sus propios intereses en las disputas políticas a nivel interno.

Los tres debates elegidos del contexto particular de Chile ilustran que tanto el gobierno como la oposición y la sociedad civil pueden decidir recurrir a instancias regionales para avanzar en sus agendas y frenar las de sus opositores. En este sentido, las organizaciones regionales proveen recursos que pueden ser clave según los temas en discusión y la coyuntura política interna de un Estado. Por lo tanto, Riggirozzi y Tussie están en lo correcto cuando plantean que, en Sudamérica, el regionalismo termina siendo «un conjunto de instituciones que potencian en vez de limitar decisiones nacionales» (Riggirozzi y Tussie 2018: 9), aunque limitaciones sobre los gobiernos pueden ocurrir, según los casos, por estrategias adoptadas por actores de la oposición o de la sociedad civil. Una consecuencia de aquello es que, finalmente, a ningún actor le conviene que las organizaciones regionales desaparezcan, pero al mismo tiempo a nadie le conviene que tengan mucho poder y autonomía.

¿Es Chile un caso excepcional? Si bien es cierto que la política de inserción internacional de Chile ha seguido una serie de características que lo distinguen del resto de la región, no faltan ejemplos ajenos para ilustrar los usos políticos de las organizaciones interamericanas por parte de actores políticos de otros países la región. En Brasil, la ex presidenta Dilma Rousseff requirió a la CIDH para tratar de bloquear el proceso de destitución en su contra, a pesar de que su propio gobierno había adoptado anteriormente varias medidas para paralizar la Comisión, cuando esta interfirió a favor de grupos indígenas del Amazonas por reclamos en contra de la construcción de una represa hidroeléctrica en el estado de Pará (Cerqueira 2016). En México, las autoridades aceptaron a regañadientes 
la intervención de organismos regionales para investigar la matanza de 43 estudiantes en Iguala en el año 2014. Dicha aceptación sirvió para que el gobierno proclamara su compromiso con la justicia y una investigación que fuese independiente. Sin embargo, cuando los peritos empezaron a criticar el actuar de las autoridades y las versiones oficiales de lo ocurrido, empezaron a sufrir las críticas de las mismas. Con el cambio de gobierno, las autoridades volvieron a acercarse a la CIDH para seguir con la investigación del caso. En la vecina Guatemala, los vaivenes del gobierno de Jimmy Morales con la Comisión Internacional contra la Impunidad en Guatemala (CICIG) siguen una lógica parecida. En Panamá, el ex presidente Ricardo Martinelli (2009-2014) trató de obtener la salida de su país del Parlamento Centroamericano, por ser una «cueva de ladrones» (La Prensa 2018), pero optó por ocupar su escaño en la entidad al término de su mandato presidencial con el fin de obtener impunidad contra los descargos de los cuales era objeto.

Analizar las organizaciones regionales a través de las interacciones entre los actores políticos nacionales también permite entender la crisis actual del «regionalismo postliberal» o «posthegemónico». Dicho regionalismo coincidió con la ola de elecciones de gobiernos de izquierda principalmente en Sudamérica, lo que facilitó el avance en muchas materias relacionadas con derechos sociales y civiles, por ejemplo, en temas de género. Esa realidad no es ajena al efecto de paralización y crisis que se percibe con las instancias más sociales del regionalismo post liberal, con el debilitamiento de la UNASUR, la promoción de PROSUR y las estrategias de debilitamiento del Sistema Interamericano de Derechos Humanos ahora por parte de gobiernos de orientación liberal en lo comercial y conservadora en lo social.

\section{BiBLIOGRAFÍA}

ADLER, Emanuel. Imagined (Security) Communities: Cognitive Regions in International Relations. Millennium: Journal of International Studies, 1997, vol. 26 (2): 249-277.

ADN. Maltrato en Cread Playa Ancha: CIDH emplaza al Estado chileno a «proteger integridad de los niños», 21/03/2018: en línea: https://www.adnradio.cl/noticias/nacional/maltratoen-cread-playa-ancha-cidh-emplaza-al-estado-chileno-a-proteger-integridad-de-losninos/20180321/nota/3726679.aspx. Fecha de consulta: 23 de julio de 2019.

Aguirre Azocar, Daniel y ERLANDSEn, Matthias. La diplomacia pública digital en América Latina: desafíos y oportunidades. Revista Mexicana de Política Exterior, 2018, vol. 113: 119-139.

ALBERT, Catalina y SEPÚLVEDA, Nicolás. El uso y abuso de psicofármacos en los hogares del SENAME. Santiago: CIPER, 2016: en línea: http://ciperchile.cl/2016/04/28/el-uso-y-abuso-de-psicofarmacos-en-los-hogares-del-sename. Fecha de consulta: 16 de junio de 2017.

ALTMANN BORBÓN, Josette. Multilateralismo en América Latina: el papel del ALBA. En ALTMANN Borbón, Josette; Beirute Brealey, Tatiana y Rojas Aravena, Francisco (eds.). América Latina y el Caribe: ¿Integrados o Marginados? Buenos Aires: Teseo, CAF, FLACSO, 2011: 207-224.

ARAujo, Kathya. Estado, sujeto y sexualidad en el Chile postdictatorial. Nomadías, 2009, vol. 9: 11-39.

BACHELET, Michelle. Estoy contigo: Programa de Gobierno 2006-2010. Santiago, 2005. 
BRICEÑO RUIZ, José. ¿Regionalismo, interregionalismo o bilateralismo?: Los retos de América Latina frente a la turbulencia global. En TREMOLADA ÁLVAREZ, Eric (ed.). La cooperación internacional como alternativa a los unilateralismos. Bogotá: Universidad Externado de Colombia, 2018: 195-236.

CAN. Decisión 666: Participación de la República de Chile en los órganos, mecanismos y medidas de la Comunidad Andina, en su condición de País Miembro Asociado. Lima: Gaceta Oficial del Acuerdo de Cartagena, 2007.

CARREÑO, Camilo. Diputados Kast y Rubilar piden a la CIDH intervenir por crisis en el SENAME. La Tercera. Santiago: 23/12/2016: en línea: https://www.latercera.com/noticia/diputadoskast-rubilar-piden-la-cidh-intervenir-crWoSs-sename. Fecha de consulta: 21 de julio de 2019.

CASON, Jeffrey. The Political Economy of Integration: The Experience of Mercosur. New York: Routledge, 2011.

CERNA, Tamara. Informe de la ONU sobre niños en el SENAME critica duramente acción del Estado y de los jueces: El documento revela vulneraciones a los derechos de los menores en Chile por más de 40 años, apuntando a la violencia en los centros y mala coordinación de los ministerios. El Mercurio, 30/07/2018: en línea: https://www.emol.com/noticias/Nacional/2018/07/30/915167/Informe-de-la-ONU-sobre-ninos-en-el-Sename-critica-duramenteaccion-del-Estado-y-los-jueces-y-revela-vulneraciones-por-mas-de-40-anos.html. Fecha de consulta: 22 de julio de 2019.

CERQueIRA, Daniel. La última apuesta de Rousseff contra el 'impeachment': ¿Ironía, falta de ética o simplemente política? El País. Madrid, 12/08/2016: en línea: https://elpais.com/internacional/2016/08/12/america/1470953241_332885.html. Fecha de consulta: 14 de octubre de 2018.

CIDH. Estadísticas. En línea: http://www.oas.org/es/cidh/multimedia/estadisticas/estadisticas. html. Fecha de consulta: 20 de julio de 2019.

COOPER, Andrew; HEINE, Jorge y THAKUR, Ramesh. Introduction: The Challenges of 21st-Century Diplomacy. En COOPER, Andrew; HeIne, Jorge y THAKUR, Ramesh (eds.). The Oxford Handbook of Modern Diplomacy. Oxford: Oxford University Press, 2013: 1-31.

DABÈNE, Olivier. The Politics of Regional Integration in Latin America: Theoretical and Comparative Explanations. New York: Palgrave Macmillan, 2009.

Dides, Claudia; BENAVEnTE, Cristina y SÁEZ, Isabel. Seguimiento a la Ley 20.418: Prevención del embarazo adolescente, educación sexual y anticoncepción de emergencia. Documento $n{ }^{\circ} 1$ del Programa de Investigación para el Desarrollo y Facultad de Ciencias de la Salud. Santiago: Universidad Central-UNFPA, 2011.

EL MERCURIO. SENAME confirma la muerte de 865 niños a cargo del organismo en últimos once años, 3/10/2016: en línea: http:/www.emol.com/noticias/Nacional/2016/10/03/824831/Senameconfirma-la-muerte-de-616-ninos-a-cargo-del-organismo-en-ultimos-once-anos.html. Fecha de consulta: 3 de enero de 2018.

Evans, Peter; JACOBSON, Harold y PuTnAm, Robert (eds.). Double-Edged Diplomacy: International Bargaining and Domestic Politics. Berkeley y Los Angeles: University of California Press, 1993.

FuEnTES, Cristián. Introducción. En ENSIGNIA, Jaime; FuENTES, Cristián y FERNÁNDEZ, María de los Ángeles (eds.). La política exterior en el Chile postconcertación: ¿quo vadis? Santiago: Fundación Friedrich Ebert Stiftung y Fundación Chile Veintiuno, 2011: 15-20.

GARCÍA, Jacobo. Derrotada la iniciativa encabezada por Colombia para reformar la defensa de Derechos Humanos en la OEA: Una oleada conservadora pretendía quitar fuerza al Sistema 
Interamericano limitando el alcance de sus resoluciones. El País, 28/06/2019: en línea: https:// elpais.com/internacional/2019/06/27/america/1561672485_548623.html. Fecha de consulta: 29 de junio de 2019.

GardinI, Gian Luca y LAmBerT, Peter (eds.). Latin American Foreign Policies: Between Ideology and Pragmatism. New York: Palgrave Macmillan, 2011.

GARRETÓN, Manuel Antonio y GARRETÓN, Roberto. La democracia incompleta en Chile: la realidad tras los rankings internacionales. Revista de Ciencia Política, 2010, vol. 30 (1): 115-148.

GOBIERNO DE CHILE. Matrimonio Igualitario: un nuevo paso hacia un Chile que valora y reconoce la diversidad. Santiago: 2017: en línea: http://www.gob.cl/matrimonio-igualitario-nuevo-pasohacia-chile-valora-reconoce-la-diversidad. Fecha de consulta: 20 de diciembre de 2017.

GuZMAN, Andrew. Why LDCS Sign Treaties That Hurt Them: Explaining the Popularity of Bilateral Investment Treaties. Virginia Journal of International Law, 1997, vol. 38: 639-688.

GuZMÁN, Juan Andrés. Niños protegidos por el Estado: los estremecedores informes que el Poder Judicial mantiene ocultos. Santiago: CIPER, 2013: en línea: http://ciperchile.cl/2013/07/04/ ninos-protegidos-por-el-estado-los-estremecedores-informes-que-el-poder-judicial-mantieneocultos. Fecha de consulta: 3 de enero de 2018.

Hermann, Margaret. How Decision Units Shape Foreign Policy: A Theoretical Framework. International Studies Review, 2001, vol. 3 (2): 47-81.

HILl, Christopher. The Changing Politics of Foreign Policy. Basingstoke: Palgrave Macmillan, 2003.

HOPENHAYN, Martín (ed.). Juventud y cobesión social en Iberoamérica: un modelo para armar. Santiago: CEPAL, 2008.

HUDSON, Valerie. Foreign policy analysis: classic and contemporary theory. Lanham: Rowman \& Littlefield Publishers, 2007.

IGUALES. Matrimonio igualitario, 2019: en línea: https://www.iguales.cl/incidencia-politica/ matrimonio-igualitario. Fecha de consulta: 22 de julio de 2019.

La PRenSA. Ricardo Martinelli informa a la Corte que renunciará al Parlacen. 21/06/2018: en línea: https://www.prensa.com/judiciales/Ricardo-Martinelli-Corte-renunciara-Parlacen_0_5058994098.html. Fecha de consulta: 21 de julio de 2019.

LATINOBARÓMETRO. Informe 2018. Santiago.

LEGLER, Thomas. De la afirmación de la autonomía a la gobernanza autónoma: el reto de América Latina y el Caribe. En Rojas ARAVEnA, Francisco (ed.). Multilateralismo vs. Soberanía: La Construcción de la Comunidad de Estados Latinoamericanos y Caribeños. Buenos Aires: Teseo y FLACSO, 2011: 23-47.

MACE, Gordon; THÉRIEN, Jean-Philippe; TussIE, Diana y DABÈnE, Olivier (eds.). Summits and Regional Governance: The Americas in Comparative Perspective. New York: Routledge, 2016.

Malamud, Andrés. Presidentialist Decision Making in Latin American Foreign Policy: Examples from Regional Integration Processes. En DOMÍngueZ, Jorge y COVARRUBIAS, Ana (eds.). Routledge Handbook of Latin America in the World. New York: Routledge, 2015: 112-123.

MerKe, Federico y Reynoso, Diego. Dimensiones de política exterior en América Latina según juicio de expertos. Estudios Internacionales, 2016, vol. 185: 107-131.

MEYER, John. World Society, Institutional Theories, and the Actor. Annual Review of Sociology, 2010, vol. 36: 1-20. 
SÉBASTIEN DUBÉ Y TATIANA ZULUAGA OROZCO

EL IMPACTO DE LA INSTRUMENTALIZACIÓN DE ORGANIZACIONES LATINOAMERICANAS

EN LA INTEGRACIÓN: TRES ILUSTRACIONES DESDE CHILE

MINREL. Principios de la Política Exterior Chilena, 2017. Santiago: Ministerio de Relaciones Exteriores de Chile: en línea: http://www.minrel.gob.cl/minrel/site/artic/20080802/pags/20080802194424. html. Fecha de consulta: 22 de julio de 2017.

MINSAL, MINEDUC y SERNAM. Diagnóstico y lineamientos de política pública para la prevención del embarazo no deseado en adolescentes. Santiago: Ministerio de Salud, Ministerio de Educación y Servicio Nacional de la Mujer, 2001.

MolinA, Ramiro. Ley 20.418: historia de una ley por la dignidad de la mujer chilena. Revista Chilena de Obstetricia y Ginecología, 2013, vol. 78 (2): 79-81.

MORAVCSIK, Andrew. Introduction: Integrating International and Domestic Theories of International Bargaining. En Evans, Peter; JACOBSON, Harold y PuTNAM, Robert (eds.). Double-Edged Diplomacy: International Bargaining and Domestic Politics. Berkeley y Los Angeles: University of California Press, 1993a: 3-42.

MoraVCSIK, Andrew. Preferences and Power in the European Community: A Liberal Intergovernmentalist Approach. Journal of Common Market Studies, 1993b, vol. 31 (4): 473-524.

NOLTE, Detlef. Lo bueno, lo malo, lo feo y lo necesario: pasado, presente y futuro del regionalismo latinoamericano. Revista Uruguaya de Ciencia Política, 2019, vol. 28 (1): 133-156.

Nueva Mayoría. Chile de Todos. Programa de Gobierno Michelle Bachelet 2014-2018. Santiago: 2013.

PLANEA. Plan Andino para la Prevención del Embarazo en Adolescentes. Quito: Organismo Andino de Salud y UNFPA, 2007.

PORTALES, Carlos. Some thoughts on summit proliferation and regional governance. En MACE, Gordon; THÉRIEN, Jean-Philippe; TussIE, Diana y DABÈnE, Olivier (eds.). Summits and Regional Governance: The Americas in Comparative Perspective. New York: Routledge, 2016.

Quilodran, Federico. Chile promulga ley de divorcio. El Nuevo Herald, 8/05/2004: en línea: http://www.latinamericanstudies.org/chile/divorcio.htm. Fecha de consulta: 21 de julio de 2019.

RiggirozZi, Pía y Tussie, Diana. Claves para leer al regionalismo sudamericano: fortaleciendo el Estado, regulando el mercado, gestionando autonomía. Perspectivas Revista de Ciencias Sociales, 2018, vol. 3 (5): 6-21.

Rivarola Puntigliano, Andrés y BRICEÑO RuIZ, José (eds.). Resilience of Regionalism in Latin America and the Caribbean: Development and Autonomy. New York: Palgrave Macmillan, 2013.

ROA, Yerko. Comisión Interamericana por polémicas en Cread Playa Ancha: Menores están en grave riesgo. Biobio Chile, 21/03/2018: en línea: https://www.biobiochile.cl/noticias/nacional/ region-de-valparaiso/2018/03/21/comWoSon-interamericana-por-polemicas-en-cread-playaancha-menores-estan-en-grave-riesgo.shtml. Fecha de consulta: 22 de julio de 2019.

RodRígueZ VignOLI, Jorge. Reproducción en la Adolescencia en América Latina y el Caribe: ¿Una anomalía a escala mundial? III Congreso de la Asociación Latinoamericana de Población (ALAP). Córdoba, Argentina, 2008.

SCHOlTE, Jan Aart. Summitry, governance, and democracy. En MACE, Gordon; THÉRIEN, JeanPhilippe; TussIE, Diana y DABÈnE, Olivier (eds.). Summits and Regional Governance: The Americas in Comparative Perspective. New York: Routledge, 2016.

SEPÚLVEDA, Nicolás y GUZMÁN, Juan Andrés. El brutal informe de la pdi sobre abusos en el SENAME que permaneció oculto desde diciembre. Santiago: CIPER, 2/07/2019: en línea: https://ciperchile. 
cl/2019/07/02/el-brutal-informe-de-la-pdi-sobre-abusos-en-el-sename-que-permaneciooculto-desde-diciembre/. Fecha de consulta: 3 de julio de 2019.

SHADLEN, Kenneth. Globalisation, Power and Integration: The Political Economy of Regional and Bilateral Trade Agreements in the Americas. Journal of Development Studies, 2008, vol. 44 (1): 1-20.

Tussie, Diana. Latin America: Contrasting Motivations for Regional Projects. Review of International Studies, 2009, vol. 36 (1): 169-188.

WALKER, Stephen. Foreign Policy Analysis and Behavioral International Relations. En WALKER, Stephen.; MALICI, Akan y SCHAFER, Mark (eds.). Rethinking Foreign Policy Analysis: States, Leaders, and the Microfoundations of Behavioral International Relations. New York: Routledge, 2011.

WEHNER, Leslie. Roles and Actions of Leadership: Brazil and the South American Others. En GODEHART, Nadine y NABERS, Dirk (eds.). Regional Powers and Regional Orders. New York: Routledge, 2011: 137-154.

WeHner, Leslie. Role Expectations As Foreign Policy: South American Secondary Powers' Expectations of Brazil as a Regional Power. Foreign Policy Analysis, 2015, vol. 11 (4): 435-455.

WeHNER, Leslie y THIES, Cameron. Role Theory, Narratives, and Interpretations: The Domestic Contestation of Roles. International Studies Review, 2014, vol. 16 (3): 411-436.

WeIfFen, Brigittte. Institutional Overlap and Responses to Political Crises in South America. En SuÁreZ, Marcial; DuARTE Villa, Rafael y WeIfFEn Brigitte (eds.). Power Dynamics and Regional Security in Latin America. London: Palgrave Macmillan, 2017: 173-197. 